Return to the Manage Active Submissions page at http://spie.org/app/submissions/tasks.aspx and approve or disapprove this submission. Your manuscript will not be published without this approval. Please contact author_help@spie.org with any questions or concerns.

\title{
Single-nanoparticle detection and spectroscopy in cells using a hyperspectral darkfield imaging technique
}

Natasha Fairbairn ${ }^{\mathrm{a}}$, Rute Fernandes ${ }^{\mathrm{a}}$, Rachel Carter ${ }^{\mathrm{b}}$, Timothy J. Elliott ${ }^{\mathrm{b}}$, Antonios G. Kanaras ${ }^{\mathrm{a}}$, Otto L. Muskens ${ }^{* a}$

${ }^{a}$ Institute for Life Sciences and Faculty of Physical and Applied Sciences, University of

Southampton, Highfield SO17 1BJ, Southampton, UK

${ }^{\mathrm{b}}$ Institute for Life Sciences and Faculty of Medicine, University of Southampton, UK

\begin{abstract}
We discuss a hyperspectral darkfield microscopy technique capable of imaging single nanoparticles at biologically compatible $\left(<0.5 \mathrm{~W} / \mathrm{cm}^{2}\right)$ illumination conditions. The microscope was tested on an array of lithographically produced gold nanorod antennas and on colloidal gold nanorods deposited on a glass substrate. As a test for in-vitro imaging capabilities, we studied the uptake of gold nanorods by DC2.4 dendritic cells.
\end{abstract}

Keywords: nanoparticles, darkfield microscopy, hyperspectral, live-cell imaging

*o.muskens@soton.ac.uk; phone 44238059 3911; fax 44238059 3910; http://www.phys.soton.ac.uk/muskens

\section{INTRODUCTION}

The detection and characterization of colloidal nanoparticles is of enormous importance in a variety of applications in chemistry, nanotechnology and biomedicine [1-13]. The requirements of such applications are strongly dependent on the type of particles and the environment in which they are imaged, and may include factors such as sensitivity, selectivity, speed, and biocompatibility. The enormous success of darkfield microscopy in biomedicine is mainly due to its compromise in many of the above aspects. While more sensitive techniques are available, darkfield typically allows resolving particles with scattering cross-sections exceeding $10^{4} \mathrm{~nm}^{2}$ on a cellular background at high imaging speed. In particular it is possible to use darkfield microscopy for the detection of metallic nanoparticles, since due to their plasmonic properties, nanoparticles with dimensions larger than typically $50 \mathrm{~nm}$ scatter light strongly at a particular resonant wavelength. Detection of smaller nanoparticles is possible but requires an increasing illumination intensity since the scattering cross section scales as the 6th power of particle diameter. Such high illumination intensities are generally not favorable for in-vitro biological studies as they may cause phototoxicity and affect cellular functions. The purpose of developing the darkfield detection method described here is to minimize the illumination intensity to allow for time-lapse imaging of live cells under biologically relevant conditions.

\section{METHOD}

\subsection{Experimental setup}

A home built darkfield microscope has been used to collect the results shown in this report, which has been set up as shown in Fig. 1 [14]. Firstly, the relevant wavelength required for illuminating the sample is selected from a supercontinuum source with an acousto-optic tunable filter (AOTF). Secondly, a ring of light is produced by sending the laser beam into an optical fiber at a slight angle, thereby coupling to one mode of the fiber, which has the effect of producing a spiral of light through the fiber. The ring of light then enters the darkfield ring of the microscope objective. Light scattered from the sample is collected using a sensitive EMCCD camera (Andor Luca). The sample is mounted on a $3 \mathrm{D}$ piezo stage which allows the user to focus and move to different parts of the sample. Brightfield transmission images were produced using a white light lamp behind the sample.

In this project the darkfield microscope has been tuned to detect nanoparticles with an LSPR in the near infrared part of the spectrum. This wavelength range is the most important for biological applications, as transmission of light through biological tissue is highest in the near infrared. Both the power of the laser light and the sensitivity of the camera have a dependency on wavelength as can be seen in Figure 2. The laser power was measured with a power meter for a range of wavelengths after the light had been filtered by the AOTF. The camera sensitivity was measured by looking at a 
Return to the Manage Active Submissions page at http://spie.org/app/submissions/tasks.aspx and approve or disapprove this submission. Your manuscript will not be published without this approval. Please contact author_help@spie.org with any questions or concerns.

broadband lambertian scattering source under darkfield illumination and correcting for the already measured laser power by controlling the amplitude of the acoustic waves in the AOTF until the laser power remained constant over the wavelength range. The measured camera sensitivity agreed well with the quantum efficiency stated by the manufacturer.

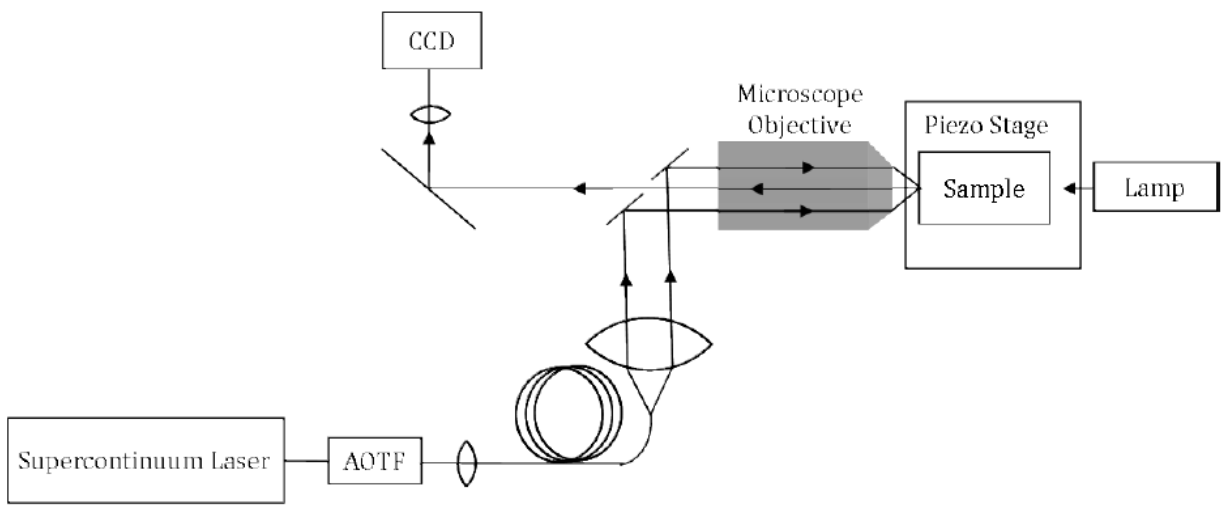

Figure 1 Experimental setup for hyperspectral darkfield microscopy.

Because of the variations in laser power and camera sensitivity with wavelength, the system needs to be well calibrated, in order to show that any change in signal seen is due to the nanoparticles, and not to the inherent changes in the sensitivity of the system at different wavelengths. A method of producing a calibration curve for the whole system is to measure the light scattered from a white paint pigment $\left(\mathrm{TiO}_{2}\right)$ sample, using the darkfield microscope, at different wavelengths. The white paint scatters equally well at all wavelengths of light in the visible range. The calibration curve produced by this method includes the camera sensitivity and laser power dependence on wavelength as well as any other possible wavelength dependencies in the system.
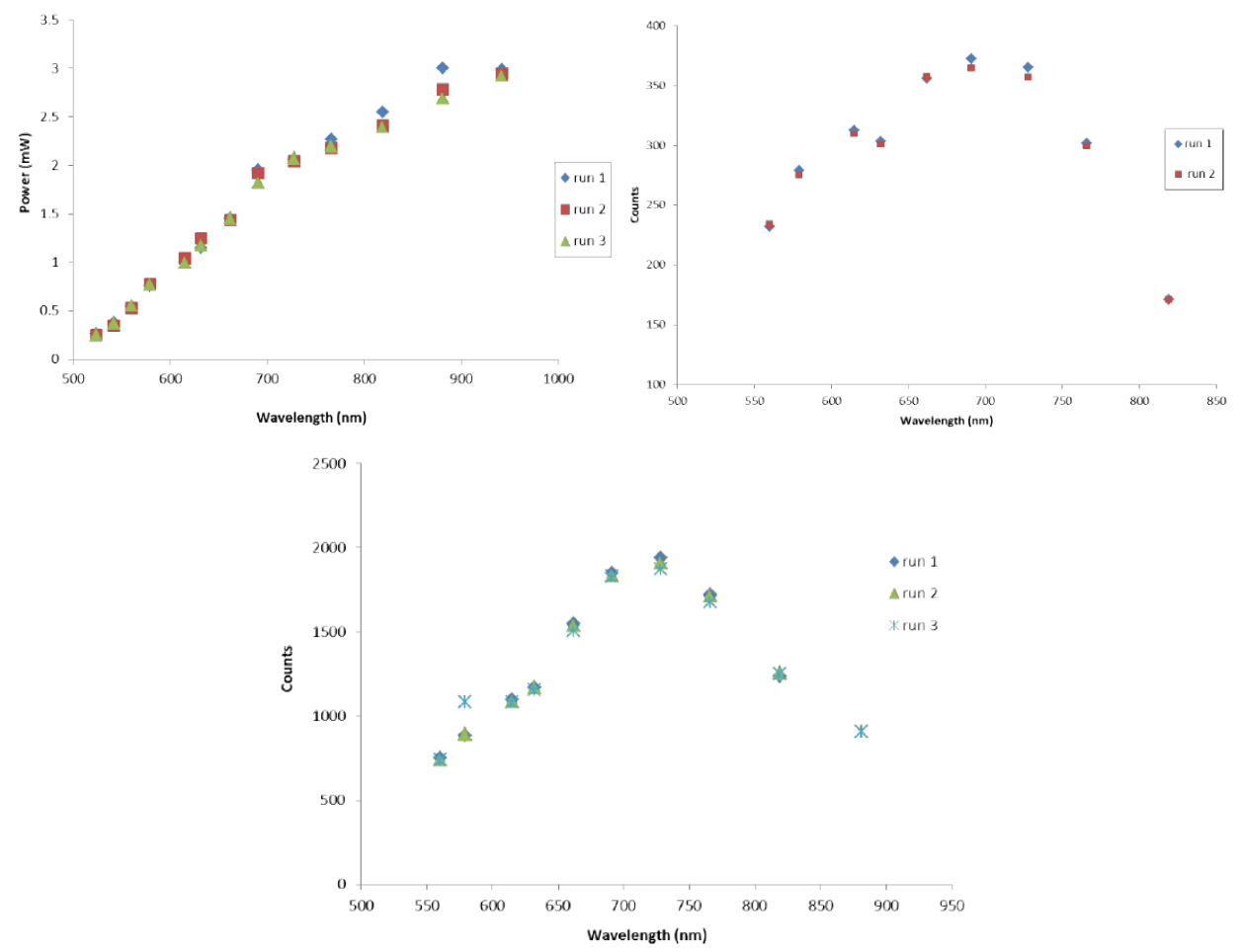

Figure 2. Calibration of the experimental setup. (top left) Output power of laser/AOTF versus wavelength, (top right) sensitivity of EMCCD camera, and (bottom) overall efficiency correction factor. 
Return to the Manage Active Submissions page at http://spie.org/app/submissions/tasks.aspx and approve or disapprove this submission. Your manuscript will not be published without this approval. Please contact author_help@spie.org with any questions or concerns.

The wavelength tunable darkfield setup can be used to collect hyperspectral information for an entire image of $1000 \times 1000$ pixels, covering an area on the sample of $145 \times 145 \mu \mathrm{m}$. The hyperspectral information is collected as follows: Using the AOTF, controlled via a Labview program, a wavelength scan of the supercontinuum light is performed and a series of images is collected. The same Labview program is used to take between three and ten images (depending on user requirement) for each wavelength of the scan with a CCD camera. From these images an averaged image is produced for each wavelength in order to reduce noise. The series of averaged images is then used in the next stage of image processing, which is performed as follows, using Matlab. First areas of interest (AOI's) are selected on the image. Next, the intensities of those AOI's are collected for each of the images in the series. Since each image in the series represents a measurement at a different wavelength, a spectrum is therefore built up for each area of interest.

The sizes of the areas of interest are set to be $7 \times 7$ pixels, an area on the sample of approximately $1 \times 1 \mu \mathrm{m}$, in order to fully include all the light from a particular nanoparticle, without including too much background signal or light from another neighboring particle. The positions of particles are also tracked from image to image using a cross correlation function, and the positions of the areas of interest are adjusted accordingly in the program. Position tracking is important, as sometimes the particles may have moved slightly from image to image due to the sample slipping, for example. A movement of the sample of about $500 \mathrm{~nm}$ is enough to move the particles partially out of their defined areas of interest, which can disrupt results.

\subsection{Preparation of dendritic cells incubated with gold nanoparticles}

Different samples were produced as follows: 1x105 DC 2.4 dendritic cells were added to each well of a 12-well microplate, each containing a coverslip and incubated for 24 hours in standard growth medium at $37^{\circ} \mathrm{C}$. Olygo ethylene glycol coated gold nanoparticle solution $(40 \mu \mathrm{l}, 20 \mu \mathrm{l}$ of concentration with longitudinal extinction peak with an optical density of 2.5) was then added to the relevant wells and centrifuged together with the cell solution (1-2mins, 250rpm), The samples were then incubated in standard growth medium at $37^{\circ} \mathrm{C}$ for periods of 1 and 2 hours. A control sample of cells without nanoparticles was also produced. The coverslips were then washed in PBS, fixed in paraformaldehyde for 7 minutes and washed again in PBS. Slides were prepared by gluing coverslips to glass slides with moviol.

\section{EXPERIMENT}

\subsection{Measurement of Longitudinal and Transverse LSPR modes of Gold Nanoantennas with Polarized Light}

The hyperspectral darkfield system has been used to measure the scattering spectra due to the longitudinal and transverse modes of a sample of plasmonic nanoantennas. The nanoantennas consist of two arms, each arm is $200 \mathrm{~nm}$ in length, $100 \mathrm{~nm}$ wide, and $25 \mathrm{~nm}$ thick with a gap between the arms of 20 to $80 \mathrm{~nm}$ [15]. In order to only collect light which has been scattered by the nanoparticle at a particular polarization angle, a polarization filter was added in front of the CCD camera. Spectra were then measured for polarization angles parallel and perpendicular to the antenna axes. Individual spectra for all nanoantennas in the array are measured simultaneously for any given polarization angle. However the spectra of six typical antennas are presented here for clarity. Figure 3(top) shows the positions of the six nanoparticles in the array. Figure 3(middle) shows the variation in light scattered by nanoantennas 1-6 with wavelength for polarization parallel $\left(0^{\circ}\right)$ and perpendicular $\left(90^{\circ}\right)$ to the antenna long axis. It is also interesting to demonstrate the difference in the polarization dependence of the nanoantennas at different wavelengths by choosing two wavelengths (the peaks of the longitudinal and transverse resonance) and scanning over polarization angle between $0^{\circ}$ and $180^{\circ}$. Figure 3 (bottom) shows how the light scattered from gold nanoantennas at different polarization angles varies in intensity due to the longitudinal and transverse plasmon resonances. It can clearly be seen that when the transmission axis of the polarizer is parallel to the long axis of the nanoantenna, there is an increase in the intensity of the $871 \mathrm{~nm}$ wavelength light. When light with a wavelength of $713 \mathrm{~nm}$ is shone onto the antennas, the measured polarization dependence shows a maximum when the transmission axis of the polarizer is parallel to the short axis of the antenna $\left(90^{\circ}\right)$.

\subsection{Detection of colloidal gold nanorods}

The hyperspectral darkfield imaging method is useful for simultaneously measuring the spectra of many nanoparticles or particle clusters on a sample. The measured spectra can also provide a good indication of whether or not the detected scattered light is due to single nanoparticles or due to a nanoparticle cluster or aggregate as the spectrum for a cluster or aggregate would be expected to be different from a single particle spectrum due to coupling effects between the nanoparticles. When polarized light is shone onto a nanoparticle, for the LSPR to be excited the direction of the 
Return to the Manage Active Submissions page at http://spie.org/app/submissions/tasks.aspx and approve or disapprove this submission. Your manuscript will not be published without this approval. Please contact author_help@spie.org with any questions or concerns.
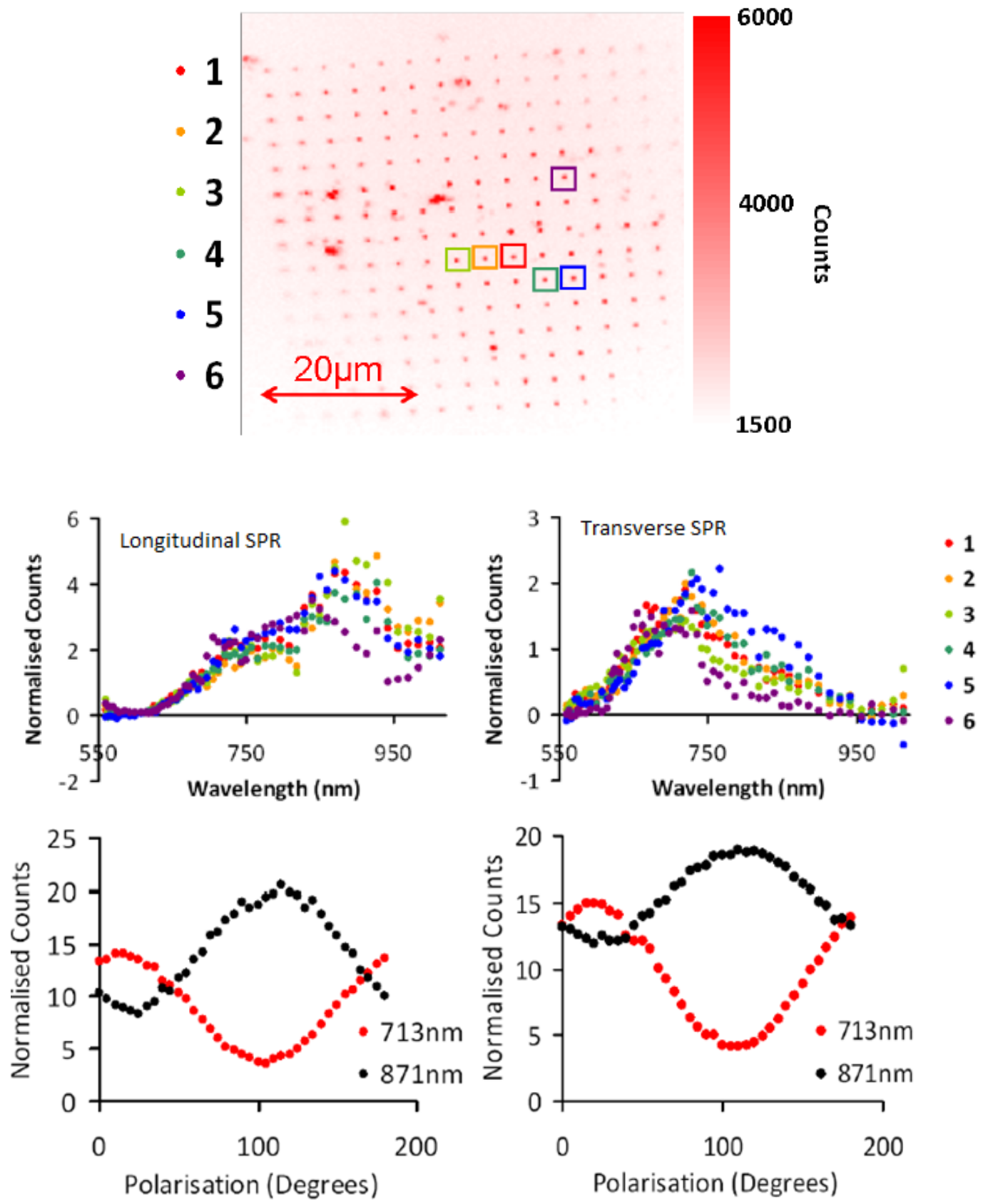

Figure 3 (top) Positions of nanoantennas for which spectra have been measured using horizontally and vertically polarized light. (middle left) Spectra of nanoantennas measured using polarized light with a transmission axis aligned with the long axis of the nanoantennas. (middle right) Spectra of nanoantennas measured with polarized light with a transmission axis aligned with the short axis of the nanoantennas. (bottom) Polarization dependence of two nanoantennas at wavelengths corresponding to the transverse and longitudinal SPR.

oscillation of the electric field of the light must match up with the direction of oscillation of electrons in the nanoparticle, required for that particular LSPR mode. If the wavelength of light is selected to match the longitudinal LSPR of a nanorod, for example, then depending on the orientation of the nanorod on the sample, a particular angle of polarization of light will excite the LSPR mode, when the angle of polarization matches up with the angle of orientation of the long axis of the nanorods. The polarization angle of light was changed and the light scattered from some parts of the sample 


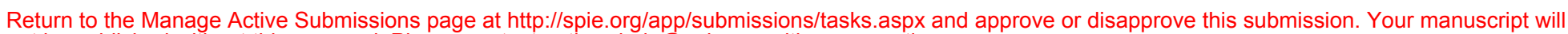
not be published without this approval. Please contact author_help@spie.org with any questions or concerns.

was observed to have a polarization dependence. An example is shown in Fig. 4 which shows two images of a sample of colloidal nanorods taken at two orthogonal polarizations. It can be observed that some bright spots that are present in the first image are not seen in the second image.

i) $0^{\circ} 632 \mathrm{~nm}$

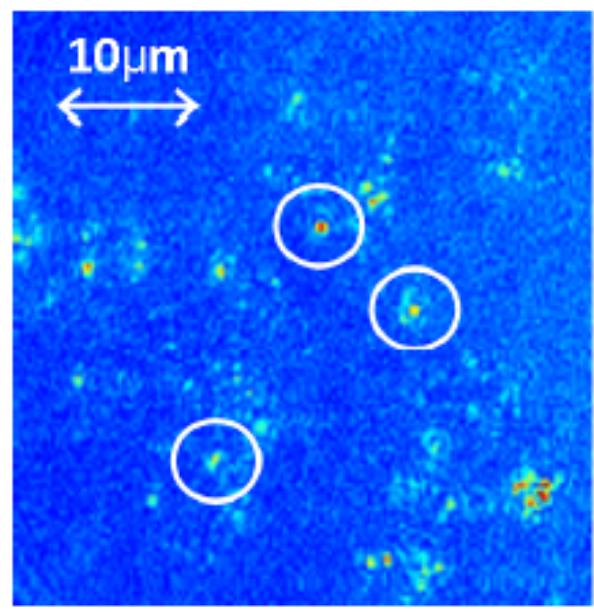

ii) $90^{\circ} 632 \mathrm{~nm}$

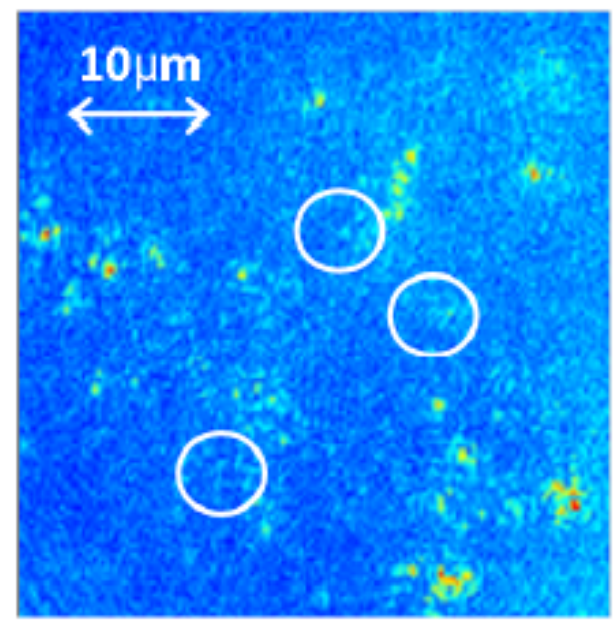

\section{Counts}

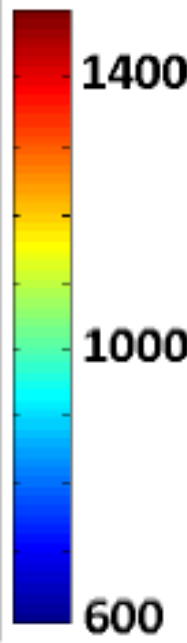

Figure 4 Darkfield image of the same area on a sample of colloidal nanorods taken at the same wavelength but at two orthogonal polarization angles..

Figure 5 shows a darkfield image and selected spectrum for a nanorod measured using the hyperspectral imaging system. The particle originates from a sample of olygoethylene glycol coated nanorods with average length $47 \mathrm{~nm}$ and average width of $16 \mathrm{~nm}$, deposited on a TEM grid. A theoretical spectrum has been calculated using a finite element method (COMSOL) for a cylindrical rod with hemispherical end caps of total length $47 \mathrm{~nm}$ (including end caps) and a diameter of $16 \mathrm{~nm}$. The height of the theoretical peak has been normalized to fit on the graph with the measured peak. The position of the measured peak agrees well with the theoretical prediction of the extinction spectrum for a single nanorod with the same dimensions as the average nanorod dimensions in the sample. The agreement of the measured peak with the theoretically predicted LSPR strongly suggests that this candidate is a single nanorod. However, it is difficult to make such a statement with surety without further information from, for example, a TEM image of the same nanoparticle.

\section{Counts}
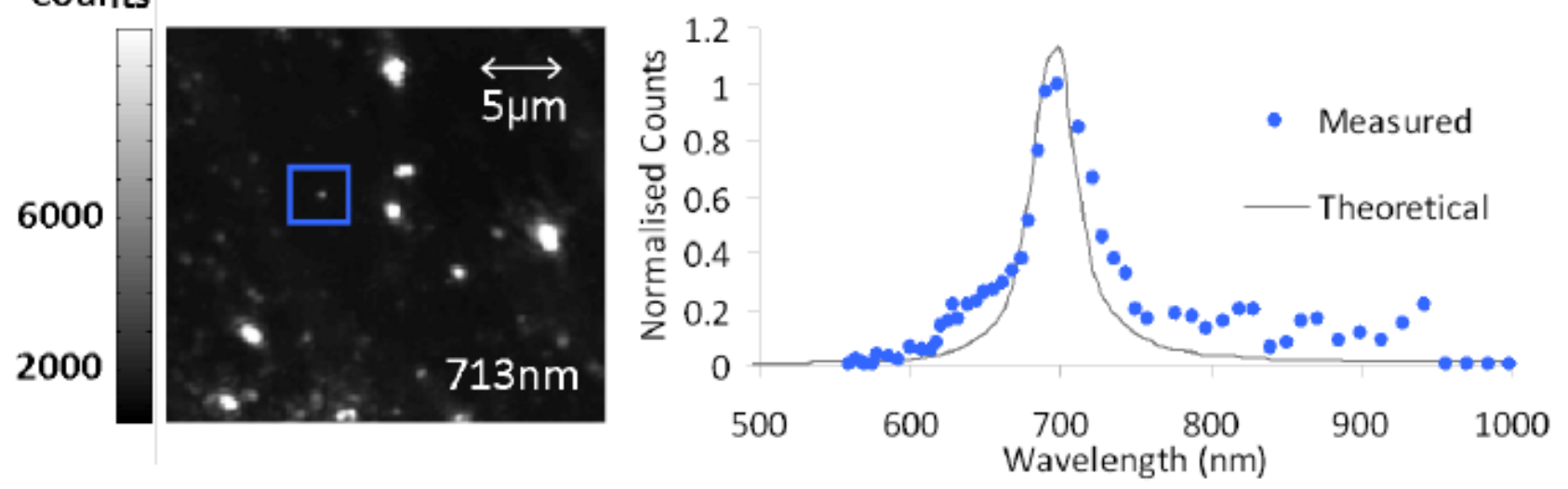

Figure 5 A darkfield image of a sample of gold nanorods taken at an illumination wavelength of $713 \mathrm{~nm}$ with a measured spectrum of the indicated bright spot on the image, shown along with a theoretically predicted spectrum (line). 


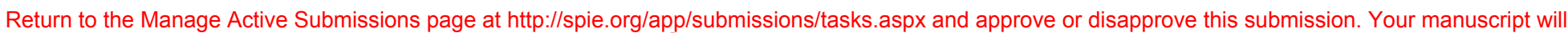
not be published without this approval. Please contact author_help@spie.org with any questions or concerns.

\subsection{Incubation of colloidal gold nanorods in dendritic cells}

Figure 6 shows dendritic cells that have been incubated with gold nanorods and a control sample of cells without nanorods. It can be clearly seen that the sample which gives the highest signal under the darkfield microscope is the sample with $40 \mu \mathrm{l}$ of nanorod solution incubated for $2 \mathrm{hrs}$, at a wavelength of $691 \mathrm{~nm}$. This wavelength is in agreement with the UV-VIS absorption spectrum as shown in Fig. 7(left). This evidence strongly suggests that the increased signal from this sample is due to the presence of nanoparticles. The spatial distribution of the detected scattering signal suggests that the nanoparticles may be mainly located inside the cell and it is likely that the nanorods have been collected in the endosomal compartments. Figure 6 shows an increase of signal for cells that have been incubated for a longer time. At higher particle loads, some strong peaks are found at longer wavelengths $(819 \mathrm{~nm})$. This effect could be due to a broadening or shift of the plasmon resonance peak originating from nanorod aggregates.

To verify that the signal observed in fig 6 originates from nanorods, the mean value of light scattered over the total area of each cell is shown for different wavelengths, corrected for system efficiency, in Fig. 7(right). In the absence of nanoparticles, a flat response is seen. Where nanoparticles are present, a clear peak is observed. Overall, the darkfield microscopy method seems to be successful at enabling detection of gold nanorods in a cellular environment as long as certain conditions are satisfied. Specifically, when the wavelength of light is at the plasmon resonance of the nanoparticles, when the concentration of nanorods is high enough, and the incubation time is long enough to allow the nanoparticles to attach to, or be taken up by the cells.

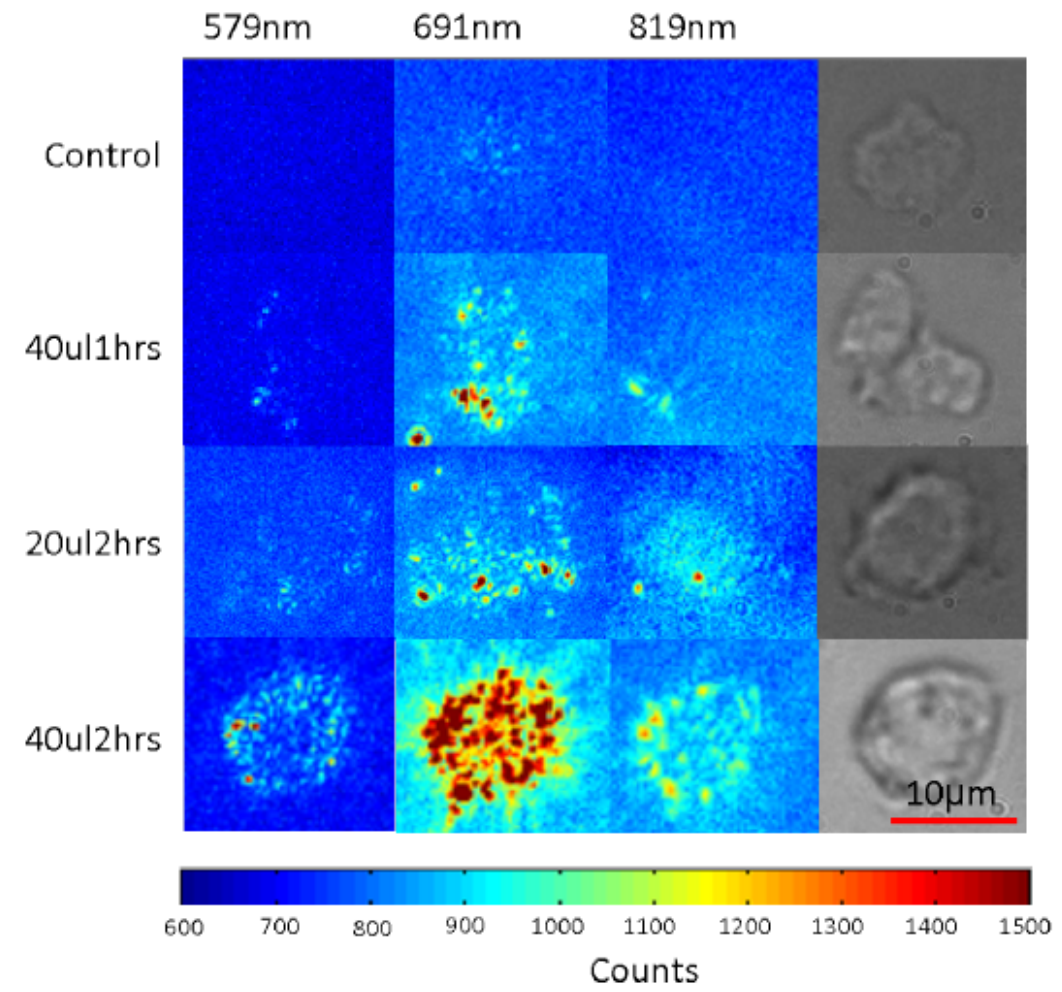

Figure 6 Summary of darkfield images from samples of dendritic cells incubated with different amounts of nanoparticles for different lengths of time. A brightfield image of each cell is also shown on the right-hand side. 
Return to the Manage Active Submissions page at http://spie.org/app/submissions/tasks.aspx and approve or disapprove this submission. Your manuscript will not be published without this approval. Please contact author_help@spie.org with any questions or concerns.
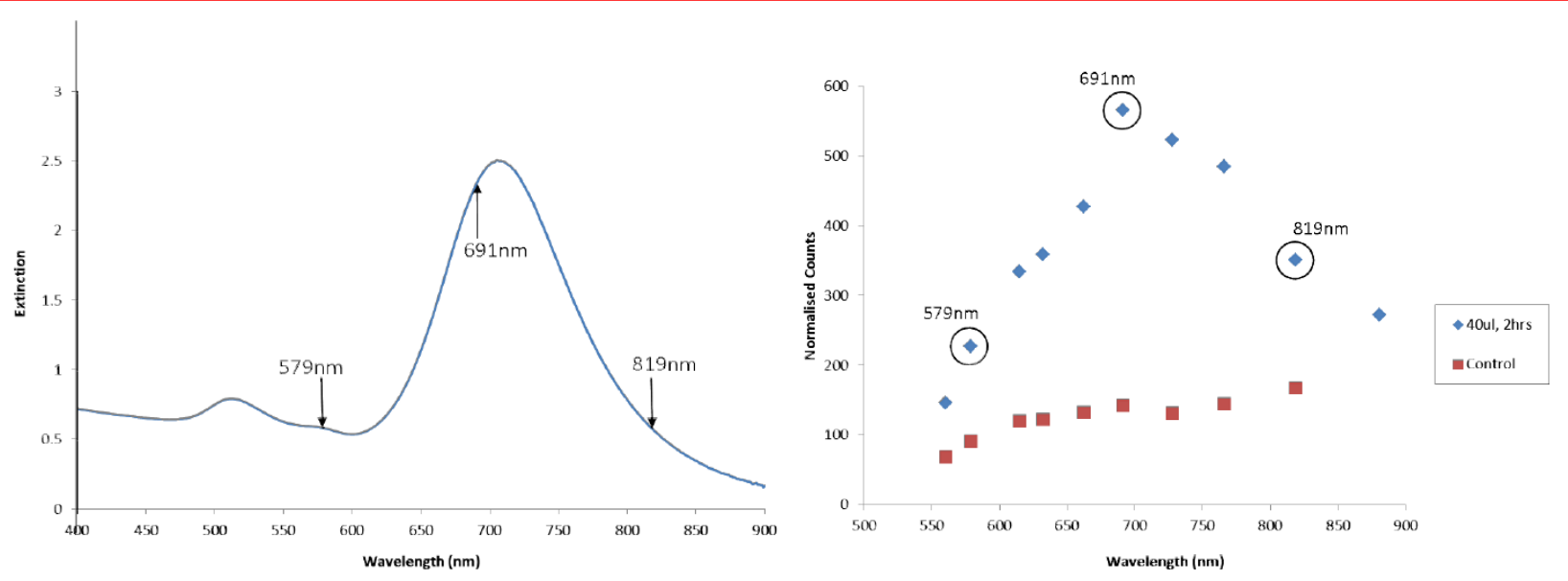

Figure 7 (a) Extinction spectrum of gold nanorod solution used in these experiments. (b) Normalized values of scattered light from sample, measured at camera, for sample containing $40 \mu$ of nanoparticles, incubated with cells for $2 \mathrm{hrs}$, and a control cell sample. Wavelengths of 579nm, 691nm, and 819nm correspond to images of Fig. 6.

\section{CONCLUSIONS}

In conclusion, the use of hyperspectral darkfield microscopy as a tool for imaging nanoparticles inside cells has been demonstrated. The wide ranging biomedical applications of nanoparticles mean that there is a high demand for methods of detecting nanoparticles on a cellular background, which can be achieved with darkfield microscopy. As a proof of principle, the detection of CTAB coated gold nanorods has been shown both on a glass substrate as well as incubated in cells. The low optical powers used translate in local intensities below $0.5 \mathrm{~W} / \mathrm{cm}^{2}$, which is low enough to limit effects of phototoxicity in live cell experiments. Future work will look at hyperspectral sensing inside cells, time-lapse studies of cellular uptake, and three-dimensional optical sectioning. Overall the approach is useful for a wide range of applications where real-time spatial and spectral information on nanoparticle systems is required.

\section{REFERENCES}

[1] Zhao, F., Zhao, Y., Liu, Y., Chang, X., Chen, C., Zha, Y., "Cellular Uptake, Intracellular Trafficking, and Cytotoxicity of Nanomaterials," Small 7, 1322-1337 (2011).

[2] Jun, Y., Sheikholeslami, S., Hostetter, D. R., Tajon, C., Craik, C. S., Alivisatos, A. P. "Continous imaging of plasmon rulers in live cells reveals early-stage caspase-3 activation at the single-molecule level," Proc. Nat. Acad. Sc. 106, 17735-17740 (2009).

[3] Acimovic, S. S., Kreuzer, M. P., Gonzalez, M. U., Quidant R., "Plasmon Near-Field Coupling in Metal Dimers as a Step toward Single-Molecule Sensing," ACS Nano 3, 1231-1237 (2009).

[4] Becker, J., Schubert, O., Sönnichsen, C., "Gold Nanoparticle Growth Monitored in situ Using a Novel Fast Optical Single-Particle Spectroscopy Method," Nano Lett. 7, 1664-1669 (2007).

[5] Podoliak, N.; Bartczak,D.; Buchnev, O. Kanaras, A. G.; Kaczmarek, M., "High Optical Nonlinearity of Nematic Liquid Crystals Doped with Gold Nanoparticles", J. Phys. Chem. C 116 (23) 12934-12939 (2012).

[6] Fairbairn, N., Light, R. A., Carter, R., Fernandes, R., Kanaras, A. G., Elliott, T. J., Somekh, M. G., Pitter, M. C., Muskens, O. L., "Spatial Modulation Microscopy for Real-Time Imaging of Plasmonic Nanoparticles and Cells," Opt. Lett. 37, 3015-3017 (2012).

[7] Schulz, K. M., Abb, S., Fernandes, R., Abb, M., Kanaras, A. G., Muskens, O. L., "Formation and Plasmonic Response of Self-Assembled Layers of Colloidal Gold Nanorods and Branched Gold Nanoparticles," Langmuir, 28, 8874-8880 (2012).

[8] McFarland, A. and Van Duyne, R., "Single Silver Nanoparticles as Real-Time Optical Sensors with Zeptomole Sensitivity," Nano Lett. 3, 1057-1062 (2003). 
Return to the Manage Active Submissions page at http://spie.org/app/submissions/tasks.aspx and approve or disapprove this submission. Your manuscript will not be published without this approval. Please contact author_help@spie.org with any questions or concerns.

[9] Bingham, J., Willets, K. A., Shah, N. C., Andrews, D. Q., Van Duyne, R. P., "Localized Surface Plasmon Resonance Imaging: Simultaneous Single Nanoparticle Spectroscopy and Diffusional Dynamics," J. Phys. Chem. C Lett. 113, 16839-16842 (2009).

[10]Zhang, L, Li, Y., Li, D.-W., Jing, C., Chen, X., Lv, M., Huang, Q., Long, Y.-T., Willner, I., "Single Gold Nanoparticles as Real-Time Optical Probes for the Detection of NADH-dependent Intracellular Metabolic Enzymatic Pathways," Angew. Chem. 50, 6789-6792 (2011).

[11] Ament, I, Prasad, J., Henkel, A., Schmachtel, S., Sonnichsen, C., "Single Unlabeled Protein Detection on Individual Plasmonic Nanoparticles; Nano Lett. 201212 p1092-1095.

[12] Mock, J., Barbic, M., Smith, D. R, Schultz, D. A., Schultz, S., "Shape effects in plasmon resonance of individual colloidal silver nanoparticles," J. Chem. Phys. 116, 6756-6759 (2002).

[13] Tamaru, H, Kuwata, H., Miyazaki, H. T., Miyano, K., "Resonant light scattering from individual Ag nanoparticles and particle pairs," App. Phys. Lett. 80, 1826-1828 (2002).

[14] Fairbairn, N., Christofidou, A., Kanaras, A. G., Newman, T. A, Muskens O. L., "Hyperspectral darkfield microscopy of single hollow gold nanoparticles for biomedical applications," Phys. Chem. Chem. Phys. DOI: 10.1039/C2CP43162A (2013).

[15] Abb, M., Albella, P., Aizpurua, J., Muskens, O. L., "All-optical control of a single plasmonic nanoantenna-ITO hybrid," Nano Lett. 11, 2457-2463 (2011). 